\title{
Rational Biparameter Homotopy Perturbation Method and Laplace-Padé Coupled Version
}

\author{
Hector Vazquez-Leal, ${ }^{1}$ Arturo Sarmiento-Reyes, ${ }^{2}$ Yasir Khan, ${ }^{3}$ \\ Uriel Filobello-Nino, ${ }^{1}$ and Alejandro Diaz-Sanchez ${ }^{2}$ \\ ${ }^{1}$ Electronic Instrumentation and Atmospheric Sciences School, University of Veracruz, Cto. Gonzalo \\ Aguirre Beltrán s/n, 91000 Xalapa, VER, Mexico \\ ${ }^{2}$ Department of Electronics, National Institute for Astrophysics, Optics and Electronics, Luis Enrique \\ Erro 1, 72840 Santa María Tonantzintla, PUE, Mexico \\ ${ }^{3}$ Department of Mathematics, Zhejiang University, Hangzhou 310027, China
}

Correspondence should be addressed to Hector Vazquez-Leal, hvazquez@uv.mx

Received 25 September 2012; Accepted 21 October 2012

Academic Editor: Chein-Shan Liu

Copyright (C) 2012 Hector Vazquez-Leal et al. This is an open access article distributed under the Creative Commons Attribution License, which permits unrestricted use, distribution, and reproduction in any medium, provided the original work is properly cited.

The fact that most of the physical phenomena are modelled by nonlinear differential equations underlines the importance of having reliable methods for solving them. This work presents the rational biparameter homotopy perturbation method (RBHPM) as a novel tool with the potential to find approximate solutions for nonlinear differential equations. The method generates the solutions in the form of a quotient of two power series of different homotopy parameters. Besides, in order to improve accuracy, we propose the Laplace-Padé rational biparameter homotopy perturbation method (LPRBHPM), when the solution is expressed as the quotient of two truncated power series. The usage of the method is illustrated with two case studies. On one side, a Ricatti nonlinear differential equation is solved and a comparison with the homotopy perturbation method (HPM) is presented. On the other side, a nonforced Van der Pol Oscillator is analysed and we compare results obtained with RBHPM, LPRBHPM, and HPM in order to conclude that the LPRBHPM and RBHPM methods generate the most accurate approximated solutions.

\section{Introduction}

Solving nonlinear differential equations is an important issue in sciences because many physical phenomena are modelled using such classes of equations. One of the most powerful methods to approximately solve nonlinear differential equations is the homotopy perturbation method (HPM) [1-45]. The HPM method is based on the use of a power series, which transforms the original nonlinear differential equation into a series of linear differential equations. In this work, we propose a generalization of the aforementioned concept by using 
a quotient of two power series of different homotopy parameters, which will be denominated as the rational biparameter homotopy perturbation method (RBHPM). In the same fashion, like HPM, the use of this quotient transforms the nonlinear differential equation into a series of linear differential equations. The generated solutions are expressed as the quotient of two truncated power series and they constitute the approximate solutions. Besides, we propose an after-treatment to the approximate solutions with the Laplace-Padé (LP) transform [46] in order to improve the accuracy of the solutions. This coupled method will be denominated as the LPRBHPM. In addition, the method is applied to two case studies, a Ricatti nonlinear differential equation [47] and a Van der Pol Oscillator [8, 48], without external forcing.

This paper is organized as follows. In Section 2, the basic idea of the RBHPM method is given. Section 3 presents a convergence analysis for the proposed method. In Section 4, the basic concept of Padé approximants is explained. In Section 5, the coupling of the RBHPM method with the Laplace-Pade transform is recast. In Section 6, the first case study, a Riccati nonlinear differential equation, is solved by using the proposed method and HPM. In Section 7, the second case study, a nonlinear oscillator problem, is treated. Section 8 is devoted to discuss the resulting solutions and some numeric analysis issues. Finally, a brief conclusion is given in Section 9.

\section{Basic Concept of RBHPM}

The RBHPM and HPM methods share common foundations. For both methods, it can be considered that a nonlinear differential equation can be expressed as

$$
A(u)-f(r)=0, \quad \text { where } r \in \Omega
$$

with the boundary condition given by

$$
B\left(u, \frac{\partial u}{\partial \eta}\right)=0, \quad \text { where } r \in \Gamma
$$

where $A$ is a general differential operator, $f(r)$ is a known analytic function, $B$ is a boundary operator, $\Gamma$ is the boundary of the domain $\Omega$, and $\partial u / \partial \eta$ denotes differentiation along the normal drawn outwards from $\Omega$ [49]. In most cases, the $A$ operator can be split into two operators, namely $L$ and $N$, which represent the linear and the nonlinear operators, respectively. Hence, (2.1) can be rewritten as

$$
L(u)+N(u)-f(r)=0 .
$$

Now, a homotopy formulation can be given as

$$
H(v, p)=(1-p)\left[L(v)-L\left(u_{0}\right)\right]+p(L(v)+N(v)-f(r))=0, \quad p \in[0,1]
$$

where $u_{0}$ is the trial function (initial approximation) for (2.3) that satisfies the boundary conditions, and $p$ is known as the perturbation homotopy parameter. 

given in

The equation above exhibits specific behaviors at the limit values $p=0$ and $p=1$, as

$$
\begin{gathered}
H(v, 0)=L(v)-L\left(u_{0}\right)=0, \\
H(v, 1)=L(v)+N(v)-f(r)=0 .
\end{gathered}
$$

For the HPM method [11-14], we assume, without loss of generality, that the solution for (2.4) can be expressed as a power series of $p$

$$
v=p^{0} v_{0}+p^{1} v_{1}+p^{2} v_{2}+\cdots
$$

In the limit, when $p \rightarrow 1$, the approximate solution for (2.1) is give as

$$
u=\lim _{p \rightarrow 1} v=v_{0}+v_{1}+v_{2}+\cdots
$$

where $v_{0}, v_{1}, v_{2}, \ldots$ are unknown functions to be determined by the HPM method. The series in (2.7) is convergent in most cases $[1,2,11,14,36]$.

For the RBHPM method, the homotopy in (2.4) can be rewritten as

$$
\begin{gathered}
H(v, p)=(1-Q)\left[L(v)-L\left(u_{0}\right)\right]+Q(L(v)+N(v)-f(r))=0, \quad p \in[0,1], q \in[0,1], \\
Q=a p+(1-a) q, \quad 0<a<1,
\end{gathered}
$$

where $p$ and $q$ are the homotopy parameters, and $a$ is a weight factor that affects them in complementary proportions.

Now, we assume that the solution for (2.8) can be written as the quotient of the power series of both homotopy parameters:

$$
v=\frac{p^{0} v_{0}+p^{1} v_{1}+p^{2} v_{2}+\cdots}{q^{0} w_{0}+q^{1} w_{1}+q^{2} w_{2}+\cdots}
$$

where $v_{0}, v_{1}, v_{2}, \ldots$ and $w_{1}, w_{2}, \ldots$ are unknown functions to be determined by the RBHPM method. In addition, $w_{0}$ is an arbitrary trial function, which is chosen in order to improve the RBHPM convergence in the same way as the trial function $u_{0}$ does for the HPM method [50].

On one side, the order of the approximation for the HPM method is determined by the highest power of $p$ considered in the formulation. On the other side, the order for the RBHPM method is given as $[i, k]$, where $i$ and $k$ are the highest power of $p$ and $q$ employed in the numerator and denominator of (2.10), respectively.

The limit of (2.10), when $p \rightarrow 1$ and $q \rightarrow 1$, provides an approximate solution for (2.3) given as

$$
u=\lim _{\substack{p \rightarrow 1 \\ q \rightarrow 1}} v=\frac{v_{0}+v_{1}+v_{2}+\cdots}{w_{0}+w_{1}+w_{2}+\cdots} .
$$


The limit above exists in the case that both limits

$$
\begin{gathered}
\lim _{p \rightarrow 1}\left(\sum_{i=0}^{\infty} v_{i}\right), \\
\lim _{q \rightarrow 1}\left(\sum_{i=0}^{\infty} w_{i}\right), \quad \text { where } \sum_{i=0}^{\infty} w_{i} \neq 0
\end{gathered}
$$

exist.

\section{Convergence of RBHPM Method}

In order to analyse the convergence of the RBHPM method, (2.8) is rewritten as

$$
L(v)=L\left(u_{0}\right)+Q\left[f(r)-N(v)-L\left(u_{0}\right)\right]=0 .
$$

After applying the inverse operator, $L^{-1}$, on both sides of (3.1), we obtain

$$
v=u_{0}+Q\left[L^{-1} f(r)-L^{-1} N(v)-u_{0}\right]
$$

By assuming that (see (2.10))

$$
v=\frac{\sum_{i=0}^{\infty} p^{i} v_{i}}{\sum_{i=0}^{\infty} q^{i} w_{i}}
$$

and after substituting (3.3) in the right-hand side of (3.2) in the following form:

$$
v=u_{0}+Q\left[L^{-1} f(r)-\left(L^{-1} N\right)\left[\frac{\sum_{i=0}^{\infty} p^{i} v_{i}}{\sum_{i=0}^{\infty} q^{i} w_{i}}\right]-u_{0}\right] .
$$

The exact solution of (2.3) is obtained in the limit when $p \rightarrow 1$ and $q \rightarrow 1$ in (3.4), which results in

$$
\begin{aligned}
u & =\lim _{\substack{p \rightarrow 1 \\
q \rightarrow 1}}\left(Q L^{-1} f(r)-Q\left(L^{-1} N\right)\left[\frac{\sum_{i=0}^{\infty} p^{i} v_{i}}{\sum_{i=0}^{\infty} q^{i} w_{i}}\right]+u_{0}-Q u_{0}\right), \\
& =L^{-1} f(r)-\left[\sum_{i=0}^{\infty}\left(L^{-1} N\right)\left(\frac{v_{i}}{\beta}\right)\right], \quad \beta=\sum_{i=0}^{\infty} w_{i},
\end{aligned}
$$

where

$$
\lim _{\substack{p \rightarrow 1 \\ q \rightarrow 1}} Q=\lim _{\substack{p \rightarrow 1 \\ q \rightarrow 1}}(a p+(1-a) q)=1
$$


In order to study the convergence of the RBHPM method, we use the Banach Theorem as reported in $[1,2,7,36]$. This theorem relates the solution of (2.3) to the fixed point problem of the nonlinear operator $N$.

Theorem 3.1 (Sufficient Condition for Convergence). Suppose that $X$ and $Y$ are Banach spaces and $N: X \rightarrow Y$ is a contractive nonlinear mapping, that is,

$$
\forall w, w^{*} \in X ; \quad\left\|N(w)-N\left(w^{*}\right)\right\| \leq \gamma\left\|w-w^{*}\right\| ; \quad 0<\gamma<1
$$

Then, according to Banach Fixed Point Theorem, $N$ has a unique fixed point $u$, that is, $N(u)=u$. Assume that the sequence generated by the RBHPM method can be written as

$$
W_{n}=N\left(W_{n-1}\right), \quad W_{n-1}=\sum_{i=0}^{n-1}\left(\frac{v_{i}}{\beta}\right), \quad n=1,2,3 \ldots
$$

and suppose that $W_{0}=v_{0} / \beta \in B_{r}(u)$, where $B_{r}(u)=\left\{w^{*} \in X \mid\left\|w^{*}-u\right\|<r\right\}$, then, under these conditions:

(i) $W_{n} \in B_{r}(u)$,

(ii) $\lim _{n \rightarrow \infty} W_{n}=u$.

Proof. (i) By inductive approach, for $n=1$ we have

$$
\left\|W_{1}-u\right\|=\left\|N\left(W_{0}\right)-N(u)\right\| \leq \gamma\left\|w_{0}-u\right\|
$$

Assuming that $\left\|W_{n-1}-u\right\| \leq \gamma^{n-1}\left\|w_{0}-u\right\|$, as induction hypothesis, then

$$
\left\|W_{n}-u\right\|=\left\|N\left(W_{n-1}\right)-N(u)\right\| \leq \gamma\left\|W_{n-1}-u\right\| \leq \gamma^{n}\left\|w_{0}-u\right\| .
$$

Using (i), we have

$$
\left\|W_{n}-u\right\| \leq r^{n}\left\|w_{0}-u\right\| \leq r^{n} r<r \Longrightarrow W_{n} \in B_{r}(u)
$$

(ii) Because of $\left\|W_{n}-u\right\| \leq r^{n}\left\|w_{0}-u\right\|$ and $\lim _{n \rightarrow \infty} \gamma^{n}=0, \lim _{n \rightarrow \infty}\left\|W_{n}-u\right\|=0$, that is,

$$
\lim _{n \rightarrow \infty} W_{n}=u .
$$




\section{Padé Approximants}

A rational approximation to $f(x)$ on $[a, b]$ is the quotient of two polynomials $P_{N}(x)$ and $Q_{M}(x)$ of degrees $N$ and $M$, respectively. We use the notation $R_{N, M}(x)$ to denote this quotient. The $R_{N, M}(x)$ Padé approximations $[51,52]$ to a function $f(x)$ are given by

$$
R_{N, M}(x)=\frac{P_{N}(x)}{Q_{M}(x)}, \quad \text { for } a \leq x \leq b .
$$

The method of Padé requires $f(x)$ and its derivative to be continuous at $x=0$. The polynomials used in (4.1) are expressed as

$$
\begin{gathered}
P_{N}(x)=p_{0}+p_{1} x+p_{2} x^{2}+\cdots+p_{N}(x) \\
Q_{M}(x)=q_{0}+q_{1} x+q_{2} x^{2}+\cdots+q_{M}(x) .
\end{gathered}
$$

The polynomials in (4.2) are constructed so that $f(x)$ and $R_{N, M}(x)$ agree at $x=0$ as well as their derivatives up to $N+M$ agree at $x=0$. A special case occurs for $Q_{0}(x)=1$, wherein the approximation in (4.1) becomes the Maclaurin expansion for $f(x)$. For a fixed value of $N+M$ the error is smallest when $P_{N}(x)$ and $Q_{M}(x)$ have the same degree or when $P_{N}(x)$ has degree one higher than $Q_{M}(x)$.

Notice that the constant coefficient of $Q_{M}$ is $q_{0}-1$. This is permissible, because it can be noted that 0 and $R_{N, M}(x)$ are not changed when both $P_{N}(x)$ and $Q_{M}(x)$ are divided by the same constant. Hence the rational function $R_{N, M}(x)$ has $N+M+1$ unknown coefficients. Assume that $f(x)$ is analytic and has the Maclaurin expansion

$$
f(x)=a_{0}+a_{1} x+a_{2} x^{2}+\cdots+a_{k} x^{k}+\cdots .
$$

And from the difference $f(x) Q_{M}(x)-P_{N}(x)=Z(x)$

$$
\left\lceil\sum_{i=0}^{\infty} a_{i} x^{i}\right\rceil\left\lceil\sum_{i=0}^{M} q_{i} x^{i}\right\rceil-\left\lceil\sum_{i=0}^{N} p_{i} x^{i}\right\rceil=\left\lceil\sum_{i=N+M+1}^{\infty} c_{i} x^{i}\right\rceil .
$$

The lower index $j=N+M+1$ in the summation on the right side of (4.4) is chosen because the first $N+M$ derivatives of $f(X)$ and $R_{N, M}(x)$ should agree at $x=0$.

When the left side of (4.4) is multiplied out and the coefficients of the powers of $x^{i}$ are set equal to zero for $k=0,1,2, \ldots, N+M$, the result is a system of $N+M+1$ linear equations:

$$
\begin{gathered}
a_{0}-p_{0}=0, \\
q_{1} a_{0}+a_{1}-p_{1}=0, \\
q_{2} a_{0}+q_{1} a_{1}+a_{2}-p_{2}=0,
\end{gathered}
$$




$$
\begin{gathered}
q_{3} a_{0}+q_{2} a_{1}+q_{1} a_{2}+a_{2}-p_{3}=0, \\
q_{M} a_{N-M}\left|q_{M-1} a_{N-M-1}\right| a_{N}-p_{N}=0, \\
q_{M} a_{N-M+1}+q_{M-1} a_{N-M+2}+\cdots+q_{1} a_{N}+a_{N+2}=0, \\
q_{M} a_{N-M+2}+q_{M-1} a_{N-M+3}+\cdots+q_{1} a_{N+1}+a_{N+3}=0, \\
\vdots \\
q_{M} a_{N}+q_{M-1} a_{N+1}+\cdots+q_{1} a_{N+M+1}+a_{N+M}=0 .
\end{gathered}
$$

Notice that the sum of the subscripts of the terms of each product is the same in each equation, and it increases consecutively from 0 to $N+M$. The $M$ equations in (4.6) involve only the unknowns $q_{1}, q_{2}, \ldots, q_{M}$ and must be firstly solved. Then the equations in (4.5) are used successively to find $p_{1}, p_{2}, \ldots, p_{N}[51]$.

\section{Laplace-Padé Transform and RBHPM Method Coupling}

The coupling of Laplace transform and Padé approximant [46] is used in order to recover part of the lost information due to the truncated power series [51, 53-60]. The process can be recast as follows.

(1) First, Laplace transformation is applied to power series.

(2) Next, $s$ is substituted by $1 / x$ in the resulting equation.

(3) After that, we convert the transformed series into a meromorphic function by forming its Padé approximant of order $[N / M]$. $N$ and $M$ are arbitrarily chosen, but they should be of smaller value than the order of the power series. In this step, the Padé approximant extends the domain of the truncated series solution to obtain better accuracy and convergence.

(4) Then, $x$ is substituted by $1 / s$.

(5) Finally, by using the inverse Laplace $s$ transformation, we obtain the modified approximate solution.

In order to improve the approximate rational solutions generated by the RBHPM method, we propose to apply the Laplace-Padé method, separately, to the denominator and the numerator in (2.11), only when they are expressed as power series. We will denominate to this process as the Laplace-Padé rational biparameter homotopy perturbation method (LPRBHPM).

\section{Case Study 1: Riccati Nonlinear Differential Equation}

Consider the Riccati nonlinear differential equation $[7,47]$

$$
y^{\prime}(x)-y(x)^{2}+1=0, \quad y(0)=0,
$$


having the exact solution given as

$$
y(x)=-\tanh (x)
$$

\subsection{Solution Calculated by RBHPM}

We establish the following homotopy equation:

$$
(1-(a p+(1-a) q))\left(L(v)-L\left(u_{0}\right)\right)+(a p+(1-a) q)\left(v^{\prime}-v^{2}+1\right)=0,
$$

where we have chosen $a=0.25$. Besides, the linear operator $L$ is given as

$$
L(v)=v^{\prime}+v+1
$$

and the trial function is

$$
u_{0}=-1+\exp (-x)
$$

We suppose that the solution of (6.3) is of order [2,2], which is expressed as follows

$$
v=\frac{v_{0}+v_{1} p+v_{2} p^{2}}{w_{0}+w_{1} q+w_{2} q^{2}}
$$

where the trial $w$-function is chosen as $w_{0}=1$.

After substituting (6.6) into (6.3), regrouping, and equating the terms having the following powers: $p^{0} q^{0}, p^{1}, p^{2}, q^{1}$, and $q^{2}$, it can be solved for $v_{0}, v_{1}, v_{2}, w_{1}$, and $w_{2}$. In order to fulfil the initial conditions of $(6.1)$, it follows that $v_{0}(0)=0, v_{1}(0)=0, v_{2}(0)=0, w_{1}(0)=0$, and $w_{2}(0)=0$.

The results are recast in the following system of differential equations:

$$
\begin{aligned}
& p^{0} q^{0}: w_{0} v_{0}^{\prime}+v_{0} w_{0}-v_{0} w_{0}^{\prime}+w_{0}^{2}=0, \quad v_{0}(0)=0, \\
& p^{1}: w_{0} v_{1}^{\prime}+v_{1} w_{0}-v_{1} w_{0}^{\prime}-a v_{0} w_{0}-a v_{0}^{2}=0, \quad v_{1}(0)=0, \\
& p^{2}: w_{0} v_{2}^{\prime}-2 a v_{0} v_{1}+v_{2} w_{0}-v_{2} w_{0}^{\prime}-a v_{1} w_{0}=0, \quad v_{2}(0)=0, \\
& q^{1}:-v_{0} w_{1}^{\prime}-v_{0} w_{0}-v_{0}^{2}+2 w_{0} w_{1}+v_{0}^{\prime} w_{1}+a v_{0}^{2}+a v_{0} w_{0}+v_{0} w_{1}=0, \quad w_{1}(0)=0, \\
& q^{2}:-v_{0} w_{2}^{\prime}+2 w_{0} w_{2}+a v_{0} w_{1}+w_{1}^{2}+v_{0} w_{2}+v_{0}^{\prime} w_{2}-v_{0} w_{1}=0, \quad w_{2}(0)=0 .
\end{aligned}
$$

Solving (6.7) yields

$$
\begin{aligned}
& v_{0}=-1+\exp (-x) \\
& v_{1}=a(-x-\exp (-x)+1) \exp (-x),
\end{aligned}
$$




$$
\begin{aligned}
& v_{2}=-a^{2}\left(-\frac{1}{2} x^{2}+\exp (-x)+x-2 x \exp (-x)-\exp (-2 x)\right) \exp (-x), \\
& w_{1}=\frac{(1-a)(-x-\exp (-x))-a+1}{\exp (x)-1}, \\
& w_{2}= \begin{cases}0, & x=0, \\
\frac{((x-2) \exp (x)+x+2)(-1+a)^{2} x}{2(\exp (x)-1)^{2}}, & x \neq 0 .\end{cases}
\end{aligned}
$$
obtain

By substituting (6.8) into (6.6), and calculating the limits when $p \rightarrow 1$ and $q \rightarrow 1$, we

$$
y(x)=\lim _{\substack{p \rightarrow 1 \\ q \rightarrow 1}} v=\frac{v_{0}+v_{1}+v_{2}}{w_{0}+w_{1}+w_{2}} .
$$

\subsection{Solution by HPM}

We establish the following homotopy equation:

$$
(1-p)\left(L(v)-L\left(u_{0}\right)\right)+p\left(v^{\prime}-v^{2}+1\right)=0
$$

where the linear operator $L$ and the trial function $u_{0}$ are (6.4) and (6.5), respectively.

Substituting (2.6) into (6.10), regrouping, and equating the terms with identical powers of $p$, it can be solved for $v_{0}, v_{1}, v_{2}$, and so on (in order to fulfil initial conditions from $v(0)=y(0)=0$, it follows that $v_{0}(0)=0, v_{1}(0)=0, v_{2}(0)=0$ and so on).

The result is recast in the following system of differential equations:

$$
\begin{aligned}
& p^{0}: v_{0}^{\prime}+v_{0}+1=0, \quad v_{0}(0)=0, \\
& p^{1}: v_{1}^{\prime}+v_{1}-v_{0}^{2}-v_{0}=0, \quad v_{1}(0)=0 \\
& p^{2}: v_{2}^{\prime}+v_{2}-2 v_{0} v_{1}-v_{1}=0, \quad v_{2}(0)=0 \\
& p^{3}: v_{3}^{\prime}+v_{3}-v_{1}^{2}-v_{2}-2 v_{0} v_{2}=0, \quad v_{3}(0)=0 \\
& p^{4}: v_{4}^{\prime}+v_{4}-2 v_{1} v_{2}-v_{3}-2 v_{0} v_{3}=0, \quad v_{4}(0)=0
\end{aligned}
$$

By solving (6.11), we obtain

$$
\begin{aligned}
& v_{0}=-1+\exp (-x), \\
& v_{1}=-(x+\exp (-x)-1) \exp (-x), \\
& v_{2}=\frac{1}{2}\left((4 x-2) \exp (-x)+2 \exp (-2 x)+x^{2}-2 x\right) \exp (-x),
\end{aligned}
$$




$$
\begin{gathered}
v_{3}=-\frac{1}{6}\left(-12 x \exp (-x)+x^{3}-3 x^{2}-6 \exp (-2 x)+12 x^{2} \exp (-x)\right. \\
\quad+18 x \exp (-2 x)+6 \exp (-3 x)) \exp (-x), \\
\begin{array}{c}
v_{4}=\frac{1}{24}\left(-4 x^{3}-48 x^{2} \exp (-x)-72 x \exp (-2 x)-24 \exp (-3 x)\right. \\
\left.+x^{4}+96 x \exp (-3 x)+108 x^{2} \exp (-2 x)+32 x^{3} \exp (-x)+24 \exp (-4 x)\right) \exp (-x), \\
\vdots
\end{array}
\end{gathered}
$$

By using (6.12) and (2.6) and calculating the limit when $p \rightarrow 1$, we obtain the second and four order approximations

$$
\begin{gathered}
y(x)=\lim _{p \rightarrow 1}\left(\sum_{i=0}^{2} v_{i} p^{i}\right)=v_{0}+v_{1}+v_{2}, \\
y(x)=\lim _{p \rightarrow 1}\left(\sum_{i=0}^{4} v_{i} p^{i}\right)=v_{0}+v_{1}+v_{2}+v_{3}+v_{4},
\end{gathered}
$$

respectively.

\section{Case Study 2: Van Der Pol Oscillator}

Consider the Van der Pol Oscillator problem $[8,48]$ without external forcing

$$
u^{\prime \prime}+u^{\prime}+u+u^{2} u^{\prime}=0, \quad u(0)=0, \quad u^{\prime}(0)=1,
$$

\subsection{Solution by the RBHPM Method}

We establish the following homotopy equation:

$$
(1-(a p+(1-a) q))\left(L(v)-L\left(u_{0}\right)\right)+(a p+(1-a) q)\left(v^{\prime \prime}+v^{\prime}+v+v^{2} v^{\prime}\right)=0,
$$

where the linear operator $L$ is

$$
L(v)=v^{\prime \prime},
$$

and the trial function is

$$
u_{0}=t .
$$

We assume that the solution for $(7.2)$ is order $[2,2]$, which is expressed as follows:

$$
v=\frac{v_{0}+v_{1} p+v_{2} p^{2}}{w_{0}+w_{1} q+w_{2} q^{2}}
$$

where the trial $w$-function is chosen as $w_{0}=1$. 
Substituting (7.5) into (7.2), regrouping, and equating the terms having the following powers: $p^{0} q^{0}, p^{1}, p^{2}, q^{1}$, and $q^{2}$, it can be solved for $v_{0}, v_{1}, v_{2}, w_{1}$, and $w_{2}$. In order to fulfil the initial conditions of (7.1), it follows that $v_{0}(0)=0, v_{0}^{\prime}(0)=1$ and the rest are $v_{i}(0)=0$, $v_{i}^{\prime}(0)=0, w_{i}(0)=0$ and $w_{i}^{\prime}(0)=0,(i=1,2,3, \ldots)$. Then, considering that $w_{0}=1$, we establish the following system:

$$
\begin{aligned}
& p^{0}: v_{0}^{\prime \prime}=0, \quad v_{0}(0)=0, \quad v_{0}^{\prime}(0)=1, \\
& p^{1}: v_{1}^{\prime \prime}+a v_{0}^{2} v_{0}^{\prime}+a v_{0}+a v_{0}^{\prime}=0, \quad v_{1}(0)=0, \quad v_{1}^{\prime}(0)=0, \\
& p^{2}: v_{2}^{\prime \prime}+a v_{0}^{2} v_{1}^{\prime}+a v_{1}^{\prime}+2 a v_{0} v_{1} v_{0}^{\prime}+a v_{1}=0, \quad v_{2}(0)=0, \quad v_{2}^{\prime}(0)=0, \\
& q^{1}:-v_{0} w_{1}^{\prime \prime}+3 v_{0}^{\prime \prime} w_{1}+v_{0}^{\prime}+v_{0}-2 v_{0}^{\prime} w_{1}^{\prime}-a v_{0}^{2} v_{0}^{\prime} \\
& \quad-a v_{0}-a v_{0}^{\prime}+v_{0}^{2} v_{0}^{\prime}=0, \quad w_{1}(0)=0, \quad w_{1}^{\prime}(0)=0, \\
& q^{2}:-v_{0} w_{2}^{\prime \prime}+v_{0}^{2} v_{0}^{\prime} w_{1}+a v_{0}^{3} w_{1}^{\prime}-v_{0}^{3} w_{1}^{\prime}-a v_{0}^{2} v_{0}^{\prime} w_{1}+a v_{0} w_{1}^{\prime}-3 a v_{0} w_{1}-4 v_{0}^{\prime} w_{1}^{\prime} w_{1} \\
& -2 v_{0} w_{1}^{\prime \prime} w_{1}-3 a v_{0}^{\prime} w_{1}-2 v_{0}^{\prime} w_{2}^{\prime}+2 v_{0}\left(w_{1}^{\prime}\right)^{2}+3 v_{0} w_{1}+3 v_{0}^{\prime} w_{1} \\
& +3 v_{0}^{\prime \prime} w_{1}^{2}-v_{0} w_{1}^{\prime}+3 v_{0}^{\prime \prime} w_{2}=0, \quad w_{2}(0)=0, \quad w_{2}^{\prime}(0)=0 .
\end{aligned}
$$

By solving (7.6), we obtain

$$
\begin{aligned}
v_{0}= & t \\
v_{1}= & -\frac{1}{12} a t^{2}\left(t^{2}+2 t+6\right) \\
v_{2}= & \frac{1}{2520} a^{2} t^{3}\left(30 t^{4}+77 t^{3}+315 t^{2}+210 t+420\right), \\
w_{1}= & \frac{1}{12}(1-a) t^{3}+\frac{1}{6}(1-a) t^{2}-\frac{1}{2} a t+k_{1} \\
w_{2}= & -\frac{5}{1008}(-1+a)^{2} t^{6}-\frac{1}{360}(-1+a)^{2} t^{5} \\
& +\frac{1}{5040}\left(-28 a+98-70 a^{2}\right) t^{4}+\frac{1}{12}(-1+a)\left(a-\frac{1}{6}+k_{1}\right) t^{3} \\
& +\frac{1}{5040}\left(420 a^{2}+\left(-840-840 k_{1}\right) a+840 k_{1}\right) t^{2}-\frac{1}{2} k_{1}(a-3) t+k_{2},
\end{aligned}
$$

where $k_{1}$ and $k_{2}$ are integration constants.

Substituting (7.7) into (7.5), and calculating the limits when $p \rightarrow 1$ and $q \rightarrow 1$, we obtain

$$
u(t)=\lim _{\substack{p \rightarrow 1 \\ q \rightarrow 1}} v=\frac{v_{0}+v_{1}+v_{2}}{w_{0}+w_{1}+w_{2}} .
$$


We select the parameters as $a=27 / 100, k_{1}=22 / 53$, and $k_{2}=-17 / 39$ by using the numerical procedure reported in [35-37].

In order to guarantee the validity of the approximate solution (7.8) for large $t$, the quotient of series solutions is transformed by the Laplace-Padé after-treatment. The procedure is applied separately to numerator and denominator of the expression in (7.8). First, Laplace transformation is applied to numerator of (7.8) and then $1 / t$ is written in place of $s$ in the equation. Then, the Padé approximant [3/3] is applied and $1 / s$ is written in place of $t$. Finally, by using the inverse Laplace $s$ transformation, we obtain the modified approximate solution for numerator

$$
\begin{aligned}
u_{n}(t)= & -0.0566603453624 \exp (-1.71426194764 t) \\
& +0.0566603453622 \exp (-0.0402536974457 t) \cos (0.736244931229 t) \\
& +1.22941439189 \exp (-0.0402536974457 t) \sin (0.736244931229 t)
\end{aligned}
$$

Now, we repeat the same process to the denominator by changing the order of the Padé approximant to [3/4], to obtain

$$
\begin{aligned}
u_{d}(t)= & 0.003937675067 \exp (-0.535832417112 t) \cos (2.71442186582 t) \\
& +0.003048633725 \exp (-0.535832417112 t) \sin (2.71442186582 t) \\
& +0.228229226307 \exp (-0.192147218554 t)+0.747030002351 \exp (0.628210432031 t)
\end{aligned}
$$

Then, the approximate solution calculated by LPRBHPM is

$$
u(t)=\frac{u_{n}(t)}{u_{d}(t)}
$$

\subsection{Solution by HPM}

We establish the following homotopy equation:

$$
(1-p)\left(L(v)-L\left(u_{0}\right)\right)+p\left(v^{\prime \prime}+v^{\prime}+v+v^{2} v^{\prime}\right)=0,
$$

where the linear operator $L$ and the trial function $u_{0}$ are (7.3) and (7.4), respectively.

Substituting (2.6) into (7.12), regrouping, and equating the terms with identical powers of $p$. In order to fulfil initial conditions of $(7.1)$, it follows that $v_{0}(0)=0, v_{0}^{\prime}(0)=1$, 
and the rest are $v_{i}(0)=0$ and $v_{i}^{\prime}(0)=0(i=1,2,3, \ldots)$. Then, we establish the following system:

$$
\begin{aligned}
& p^{0}: v_{0}^{\prime \prime}=0, \quad v_{0}(0)=0, \quad v_{0}^{\prime}(0)=1, \\
& p^{1}: v_{1}^{\prime \prime}+v_{0}+v_{0}^{2} v_{0}^{\prime}+v_{0}^{\prime}=0, \quad v_{1}(0)=0, \quad v_{1}^{\prime}(0)=0, \\
& p^{2}: v_{2}^{\prime \prime}+v_{0}^{2} v_{1}^{\prime}+v_{1}+2 v_{0} v_{1} v_{0}^{\prime}+v_{1}^{\prime}=0, \quad v_{2}(0)=0, \quad v_{2}^{\prime}(0)=0, \\
& p^{3}: v_{3}^{\prime \prime}+v_{0}^{2} v_{2}^{\prime}+2 v_{0} v_{1} v_{1}^{\prime}+v_{1}^{2} v_{0}^{\prime}+v_{2}^{\prime}+v_{2}+2 v_{0} v_{2} v_{0}^{\prime}=0, \quad v_{3}(0)=0, \quad v_{3}^{\prime}(0)=0, \\
& p^{4}: v_{4}^{\prime \prime}+2 v_{1} v_{2} v_{0}^{\prime}+v_{1}^{2} v_{1}^{\prime}+2 v_{0} v_{3} v_{0}^{\prime}+v_{3}^{\prime}+v_{0}^{2} v_{3}^{\prime} \\
& \quad+2 v_{0} v_{1} v_{2}^{\prime}+2 v_{0} v_{2} v_{1}^{\prime}+v_{3}=0, \quad v_{4}(0)=0, \quad v_{4}^{\prime}(0)=0 .
\end{aligned}
$$

By solving (7.13), we obtain

$$
\begin{aligned}
v_{0}= & t, \\
v_{1}= & -\frac{1}{12} t^{4}-\frac{1}{6} t^{3}-\frac{1}{2} t^{2}, \\
v_{2}= & \frac{1}{84} t^{7}+\frac{11}{360} t^{6}+\frac{1}{8} t^{5}+\frac{1}{12} t^{4}+\frac{1}{6} t^{3}, \\
v_{3}= & -\frac{19}{10080} t^{10}-\frac{67}{10080} t^{9}-\frac{53}{1680} t^{8}-\frac{31}{720} t^{7}-\frac{67}{720} t^{6}-\frac{1}{40} t^{5}-\frac{1}{24} t^{4}, \\
v_{4}= & \frac{737}{2358720} t^{13}+\frac{1889}{1330560} t^{12}+\frac{8347}{1108800} t^{11}+\frac{14039}{907200} t^{10} \\
& +\frac{13921}{362880} t^{9}+\frac{11}{336} t^{8}+\frac{25}{504} t^{7}+\frac{1}{180} t^{6}+\frac{1}{120} t^{5} .
\end{aligned}
$$

By substituting the solutions from (7.14) into (2.6) and calculating the limit when $p \rightarrow$ 1 , we can obtain the second order approximation

$$
u(t)=\lim _{p \rightarrow 1}\left(\sum_{i=0}^{2} v_{i} p^{i}\right)=t-\frac{1}{2} t^{2}+\frac{1}{84} t^{7}+\frac{11}{360} t^{6}+\frac{1}{8} t^{5} .
$$

In the same way, we obtain the four order approximation

$$
\begin{aligned}
u(t)=\lim _{p \rightarrow 1}\left(\sum_{i=0}^{4} v_{i} p^{i}\right)= & t-\frac{1}{2} t^{2}+\frac{31}{1680} t^{7}-\frac{41}{720} t^{6}+\frac{13}{120} t^{5}+\frac{12329}{907200} t^{10} \\
& +\frac{11509}{362880} t^{9}+\frac{1}{840} t^{8}-\frac{1}{24} t^{4}+\frac{737}{2358720} t^{13} \\
& +\frac{1889}{1330560} t^{12}+\frac{8347}{1108800} t^{11}
\end{aligned}
$$




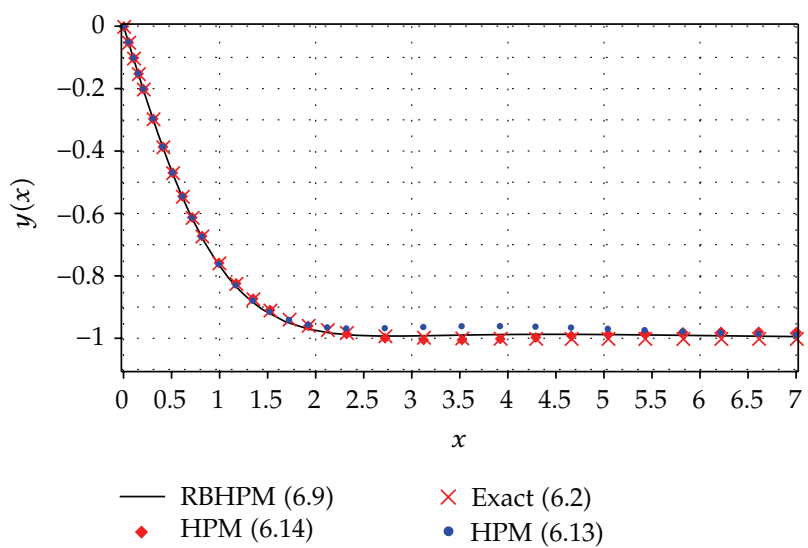

Figure 1: Exact solution (6.2) (diagonal cross) for (6.1) and its approximate solutions (6.9) (solid line), (6.13) (solid circles), and (6.14) (solid diamonds).

Table 1: Comparison between exact solution (6.2) for (6.1) to the results of approximations (6.9), (6.13), and (6.14)

\begin{tabular}{lcccc}
\hline & & Homotopy $(6.3)$ & Homotopy $(6.10)$ & Homotopy (6.10) \\
Order & Exact (6.2) & RBHPM $(6.9)$ & HPM (6.13) & HPM (6.14) \\
$x$ & 0.000000000000 & 0.000000000000 & 0.000000000000 & 0.000000000000 \\
\hline 0.00 & -0.462117157260 & -0.462720433177 & -0.462402288846 & -0.462119211588 \\
0.50 & -0.905148253645 & -0.918326771288 & -0.911212665075 & -0.90475203875 \\
1.50 & -0.964027580076 & -0.975747936207 & -0.960889970046 & -0.965901380964 \\
2.00 & -0.986614298151 & -0.991622046961 & -0.968972449805 & -0.992586779171 \\
2.50 & -0.998177897611 & -0.989972317008 & -0.961440997373 & -1.00443324553 \\
3.50 & -0.999329299739 & -0.98793701472 & -0.961349802243 & -1.0000486249 \\
4.00 & -0.999753210848 & -0.987368801973 & -0.96441914623 & -0.993370392728 \\
4.50 & -0.999909204263 & -0.988053048524 & -0.969315733164 & -0.9868655576296 \\
5.00 & -0.999966597156 & -0.98949557545 & -0.974818141376 & -0.981957532724 \\
5.50 & -0.999987711651 & -0.991249069742 & -0.980108525233 & -0.979148093068 \\
6.00 & -0.999995479351 & -0.993004075085 & -0.984752811149 & -0.97832221949 \\
6.50 & -0.999998336944 & -0.994584980578 & -0.988591496328 & -0.979053617564 \\
7.00 & A.A.E. & $8.15(-3)$ & $1.84(-2)$ & $8.93(-3)$ \\
\hline
\end{tabular}

\section{Numerical Simulation and Discussion}

On one hand, Figure 1 and Table 1 show a comparison between the exact solution (6.2) for the Riccati nonlinear differential equation (6.1) and the analytic approximations (6.9), (6.13), and (6.14). The RBHPM method of order $[2,2]$ gives the smallest average absolute error (A.A.E.) of all solutions, followed by the solutions obtained with HPM of order 4 and 2. It is remarkable to observe that the RBHPM method generates an accurate rational approximation that successfully replicates the asymptotic behaviour of (6.2).

On the other hand, Figure 2 and Table 2 show a comparison between the Fehlberg fourth-fifth order Runge-Kutta method with degree four interpolant (RKF45) [61, 62] solution (built-in function of Maple software) for the Van der Pol Oscillator (7.1) 


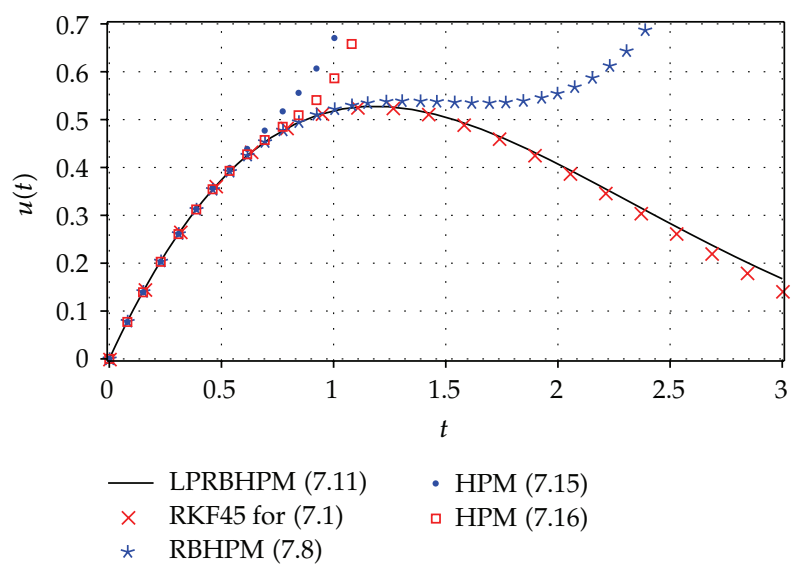

Figure 2: RKF45 solution for Van Der Pol Oscillator problem (7.1) (diagonal cross) and its approximate solutions obtained by RBHPM (7.8) (asterisk), LPRBHPM (7.11) (solid line), HPM (7.15) (solid circles), and HPM (7.16) (empty squares).

and the analytic approximations (7.8), (7.11), (7.15), and (7.16). The LPRBHPM method of order $[2,2]$ (see $(7.11)$ ) yields the smallest A.A.E. of all solutions, followed by $(7.8)$ obtained by the RBHPM method of order $[2,2]$, and finally by approximations calculated by HPM of order 4 and 2. In this case study, both LPRBHPM and RBHPM generate more accurate solutions than HPM.

The coupling of Laplace and Padé with RBHPM was a key factor for improving the accuracy in the Van der Pol problem. Both numerator and denominator of the rational expression (7.8) were considered as truncated power series. Laplace-Padé after-treatment [46] was successfully applied, and it yields better accuracy and it allows for larger ranges of the domain. Additionally, the trial functions $w_{0}$ and $u_{0}$ play an important role in the behaviour of the RBHPM and LPRBHPM methods; therefore, future research must be done in order to understand what kinds of trial functions produce better results; in this context, the operators $L$ and $N$ will be a key aspect to consider. Furthermore, it is important to point out that the RBHPM and LPRBHPM methods do not resort to linearization, a perturbation parameter, or assumptions of weak nonlinearity, and it clearly results that the generated solution has a general character and it is more realistic compared to the method of simplifying the physical problems.

Finally, the homotopy in (2.4) can be replaced by homotopy schemes such as those reported in the literature [63-93]. This future line of research can lead us to improve the performance of RBHPM or LPRBHPM methods.

\section{Conclusions}

This paper presented the RBHPM and LPRBHPM methods as a novel tool with high potential to solve nonlinear differential equations. Furthermore, a comparison between the results of applying the proposed methods and HPM was given. For the Riccati nonlinear asymptotic problem, a comparison between the RBHPM and HPM methods was presented, showing how the RBHPM method generates highly accurate approximate solutions, similar to the results obtained when using the HPM method. Additionally, a Van der Pol Oscillator 
Table 2: Comparison numerical solution (RKF45) for (7.1) and its first order approximate solutions given by RBHPM (7.8), LPRBHPM (7.11), HPM (7.15), and HPM (7.16).

\begin{tabular}{|c|c|c|c|c|c|}
\hline Order & & {$[2,2]$} & {$[2,2]$} & 2 & 4 \\
\hline$t$ & RKF45 for (7.1) & RBHPM (7.8) & LPRBHPM (7.11) & HPM (7.15) & HPM (7.16) \\
\hline 0.00 & 0.000000000000 & 0.000000000000 & $-2.04249011(-13)$ & 0.000000000000 & 0.000000000000 \\
\hline 0.10 & 0.094996749389 & 0.0963305722762 & 0.0963305680033 & 0.095001281746 & 0.0949968616125 \\
\hline 0.20 & 0.179964338949 & 0.181413957989 & 0.181413692649 & 0.180042107937 & 0.179964612584 \\
\hline 0.30 & 0.254886117960 & 0.255755918036 & 0.255752978888 & 0.255328628571 & 0.254889069792 \\
\hline 0.40 & 0.319824133934 & 0.319864038194 & 0.319847953401 & 0.321424660317 & 0.319850515572 \\
\hline 0.50 & 0.374962243912 & 0.374261968751 & 0.374202151797 & 0.379476686508 & 0.3751195795 \\
\hline 0.60 & 0.420620714410 & 0.419505461842 & 0.419331298484 & 0.431478857143 & 0.421336349604 \\
\hline 0.70 & 0.457249830610 & 0.456198011306 & 0.455769940137 & 0.480583988889 & 0.459927483684 \\
\hline 0.80 & 0.485408608033 & 0.485004250862 & 0.484075378004 & 0.531466565079 & 0.49405125938 \\
\hline 0.90 & 0.505735525317 & 0.506659764874 & 0.504828319804 & 0.590743735714 & 0.53057647436 \\
\hline 1.00 & 0.518917385708 & 0.521976574206 & 0.518630236646 & 0.66746031746 & 0.583931084348 \\
\hline 1.10 & 0.525660073662 & 0.531844221386 & 0.526097880422 & 0.773643793651 & 0.683143422756 \\
\hline 1.20 & 0.526664476070 & 0.537227026265 & 0.527855737463 & 0.924935314286 & 0.884097382101 \\
\hline 1.30 & 0.522608206722 & 0.539158647966 & 0.524527343795 & 1.14130269603 & 1.2900013214 \\
\hline 1.40 & 0.514133103782 & 0.538735520529 & 0.516726377800 & 1.44784142222 & 2.08441286596 \\
\hline 1.50 & 0.501837942107 & 0.537111006964 & 0.505048313285 & 1.87566964286 & 3.58297069111 \\
\hline 1.60 & 0.486275223293 & 0.535492254682 & 0.490063208382 & 2.4629231746 & 6.31238329453 \\
\hline 1.70 & 0.467950980699 & 0.535141785803 & 0.472309972142 & 3.25585650079 & 11.128360908 \\
\hline 1.80 & 0.447326548414 & 0.537385900716 & 0.452292231487 & 4.31005577143 & 19.3882241197 \\
\hline 1.90 & 0.424821677896 & 0.54363212026 & 0.430475743238 & 5.69176980317 & 33.1990855168 \\
\hline 2.00 & 0.400817960941 & 0.555398274173 & 0.407287171967 & 7.47936507937 & 55.769016169 \\
\hline 2.10 & 0.375662771298 & 0.574356631824 & 0.383113985298 & 9.76491075 & 91.8967515218 \\
\hline 2.20 & 0.349672525187 & 0.602397906631 & 0.358305196653 & 12.6558996317 & 148.645576503 \\
\hline 2.30 & 0.323136129463 & 0.641722425466 & 0.333172699524 & 16.2771112079 & 236.259417439 \\
\hline 2.40 & 0.296317411714 & 0.694969880802 & 0.307992974314 & 20.7726226286 & 369.394267712 \\
\hline 2.50 & 0.269457558120 & 0.765406030734 & 0.28300899685 & 26.3079737103 & 568.756347393 \\
\hline 2.60 & 0.242776634418 & 0.857196643743 & 0.258432227399 & 33.0724919365 & 863.260364544 \\
\hline 2.70 & 0.216475028642 & 0.975820118585 & 0.234444603854 & 41.2817834571 & 1292.84749061 \\
\hline 2.80 & 0.190734203918 & 1.12870912808 & 0.211200498849 & 51.1803960889 & 1912.13383476 \\
\hline 2.90 & 0.165717437177 & 1.32628670185 & 0.188828626412 & 63.0446603151 & 2795.09702573 \\
\hline \multirow[t]{2}{*}{3.00} & 0.141570064350 & 1.58371504978 & 0.167433899647 & 77.1857142857 & 4041.05178634 \\
\hline & A.A.E. & 0.231 & 0.00641 & 12.1 & 401 \\
\hline
\end{tabular}

problem was tackled with the proposed methods and compared to solutions obtained by HPM. It resulted that the RBHPM and LPRBHPM methods produced the most accurate approximated solutions. Because RBHPM, LPRBHPM, and HPM are closely related, it is possible that differential equations solved by HPM can be solved by using RBHPM or LPRBHPM. Besides, further research should be done to apply the proposed methods to the calculation of approximate solutions of nonlinear partial differential equations, nonlinear fractional equations, and boundary value problems, among others. An important remark is that the RBHPM and LPRBHPM methods do not resort to any kind of linearization procedure or perturbation parameter. Thereupon, these methods promise to become important 
mathematical tools, useful for scientist and engineers working in the area of mathematical modelling and computer simulation.

\section{Acknowledgments}

The authors gratefully acknowledge the financial support of the National Council for Science and Technology of Mexico (CONACyT) through Grant CB-2010-01 no. 157024. The authors would like to thank Roberto Castaneda-Sheissa, Rogelio-Alejandro Callejas-Molina, and Roberto Ruiz-Gomez for their contribution to this project.

\section{References}

[1] J. Biazar and H. Aminikhah, "Study of convergence of homotopy perturbation method for systems of partial differential equations," Computers $\mathcal{E}$ Mathematics with Applications, vol. 58, no. 11-12, pp. 2221-2230, 2009.

[2] J. Biazar and H. Ghazvini, "Convergence of the homotopy perturbation method for partial differential equations," Nonlinear Analysis. Real World Applications, vol. 10, no. 5, pp. 2633-2640, 2009.

[3] D. Ağırseven and T. Öziş, "An analytical study for Fisher type equations by using homotopy perturbation method," Computers \& Mathematics with Applications, vol. 60, no. 3, pp. 602-609, 2010.

[4] T. Öziş and D. Ağırseven, "He's homotopy perturbation method for solving heat-like and wave-like equations with variable coefficients," Physics Letters A, vol. 372, no. 38, pp. 5944-5950, 2008.

[5] A. Yildirim and T. Öziş, "Solutions of singular IVPs of Lane-Emden type by homotopy perturbation method," Physics Letters A, vol. 369, no. 1-2, pp. 70-76, 2007.

[6] T. Öziş and A. Yildirim, "A note on He's homotopy perturbation method for van der Pol oscillator with very strong nonlinearity," Chaos, Solitons and Fractals, vol. 34, no. 3, pp. 989-991, 2007.

[7] H. Vázquez-Leal, "Rational homotopy perturbation method," Journal of Applied Mathematics, vol. 2012, Article ID 490342, 14 pages, 2012.

[8] A. Barari, M. Omidvar, A. R. Ghotbi, and D. D. Ganji, "Application of homotopy perturbation method and variational iteration method to nonlinear oscillator differential equations," Acta Applicandae Mathematicae, vol. 104, no. 2, pp. 161-171, 2008.

[9] J. H. He, "Comparison of homotopy perturbation method and homotopy analysis method," Applied Mathematics and Computation, vol. 156, no. 2, pp. 527-539, 2004.

[10] J. H. He, "An elementary introduction to the homotopy perturbation method," Computers $\mathcal{E}$ Mathematics with Applications, vol. 57, no. 3, pp. 410-412, 2009.

[11] J. H. He, "Homotopy perturbation technique," Computer Methods in Applied Mechanics and Engineering, vol. 178, no. 3-4, pp. 257-262, 1999.

[12] J. H. He, "The homotopy perturbation method for nonlinear oscillators with discontinuities," Applied Mathematics and Computation, vol. 151, no. 1, pp. 287-292, 2004.

[13] J. H. He, "Homotopy perturbation method: a new nonlinear analytical technique," Applied Mathematics and Computation, vol. 135, no. 1, pp. 73-79, 2003.

[14] J. H. He, "Coupling method of a homotopy technique and a perturbation technique for non-linear problems," International Journal of Non-Linear Mechanics, vol. 35, no. 1, pp. 37-43, 2000.

[15] J. H. He, "Application of homotopy perturbation method to nonlinear wave equations," Chaos, Solitons and Fractals, vol. 26, no. 3, pp. 695-700, 2005.

[16] J. H. He, "Homotopy perturbation method for solving boundary value problems," Physics Letters A, vol. 350, no. 1-2, pp. 87-88, 2006.

[17] Y. Khan, H. Vazquez-Leal, and Q. Wu, "An efficient iterated method for mathematical biology model," Neural Computing and Applications. In press.

[18] J. Biazar and M. Eslami, "A new homotopy perturbation method for solving systems of partial differential equations," Computers and Mathematics with Applications, vol. 62, no. 1, pp. 225-234, 2011.

[19] J. Biazar and B. Ghanbari, "The homotopy perturbation method for solving neutral functionaldifferential equations with proportional delays," Journal of King Saud University, vol. 24, no. 1, pp. 33-37, 2012. 
[20] D. D. Ganji, H. Tari, and M. B. Jooybari, "Variational iteration method and homotopy perturbation method for nonlinear evolution equations," Computers and Mathematics with Applications, vol. 54, no. 7-8, pp. 1018-1027, 2007.

[21] M. Sheikholeslami, H. R. Ashorynejad, D. D. Ganji, and A. Yildirim, "Homotopy perturbation method for three-dimensional problem of condensation film on inclined rotating disk," Scientia Iranica, vol. 19, no. 3, pp. 437-442, 2012.

[22] D. D. Ganji and A. Sadighi, "Application of homotopy-perturbation and variational iteration methods to nonlinear heat transfer and porous media equations," Journal of Computational and Applied Mathematics, vol. 207, no. 1, pp. 24-34, 2007.

[23] D. D. Ganji, "The application of He's homotopy perturbation method to nonlinear equations arising in heat transfer," Physics Letters A, vol. 355, no. 4-5, pp. 337-341, 2006.

[24] M. Rafei, D. D. Ganji, and H. Daniali, "Solution of the epidemic model by homotopy perturbation method," Applied Mathematics and Computation, vol. 187, no. 2, pp. 1056-1062, 2007.

[25] D. D. Ganji and A. Rajabi, "Assessment of homotopy-perturbation and perturbation methods in heat radiation equations," International Communications in Heat and Mass Transfer, vol. 33, no. 3, pp. 391-400, 2006.

[26] N. Faraz and Y. Khan, "Analytical solution of electrically conducted rotating flow of a second grade fluid over a shrinking surface," Ain Shams Engineering Journal, vol. 2, no. 34, pp. 221-226, 2011.

[27] Y. Khan, Q. Wu, N. Faraz, and A. Yildirim, "The effects of variable viscosity and thermal conductivity on a thin film flow over a shrinking/stretching sheet," Computers and Mathematics with Applications, vol. 61, no. 11, pp. 3391-3399, 2011.

[28] M. Madani, M. Fathizadeh, Y. Khan, and A. Yildirim, "On the coupling of the homotopy perturbation method and Laplace transformation," Mathematical and Computer Modelling, vol. 53, no. 9-10, pp. 19371945, 2011.

[29] H. Aminikhah, "The combined laplace transform and new homotopy perturbation methods for stiff systems of odes," Applied Mathematical Modelling, vol. 36, no. 8, pp. 3638-3644, 2012.

[30] F. I. Compean, D. Olvera, F. J. Campa, L. N. Lopez de Lacalle, A. Elias-Zuniga, and C. A. Rodriguez, "Characterization and stability analysis of a multivariable milling tool by the enhanced multistage homotopy perturbation method," International Journal of Machine Tools and Manufacture, vol. 57, pp. 27-33, 2012.

[31] A. M. A. El-Sayed, A. Elsaid, I. L. El-Kalla, and D. Hammad, “A homotopy perturbation technique for solving partial differential equations of fractional order in finite domains," Applied Mathematics and Computation, vol. 218, no. 17, pp. 8329-8340, 2012.

[32] Y. Khan, H. Vazquez-Leal, and N. Faraz, "An auxiliary parameter method using adomian polynomials and laplace transformation for nonlinear differential equations," Applied Mathematical Modelling. In press.

[33] Y. Khan, H. Vazquez-Leal, and L. Hernandez-Martinez, "Removal of noise oscillation term appearing in the nonlinear equation solution," Journal of Applied Mathematics, vol. 2012, Article ID 387365, 9 pages, 2012.

[34] S. T. Mohyud-Din, A. Yildirim, and M. Inc, "Coupling of homotopy perturbation and modified Lindstedt-Poincaré methods for traveling wave solutions of the nonlinear Klein-Gordon equation," Journal of King Saud University, vol. 24, no. 2, pp. 187-191, 2012.

[35] H. Vazquez-Leal, R. Castaneda-Sheissa, U. Filobello-Nino, A. Sarmiento-Reyes, and J. Sanchez Orea, "High accurate simple approximation of normal distribution related integrals," Mathematical Problems in Engineering, vol. 2012, Article ID 124029, 22 pages, 2012.

[36] H. Vázquez-Leal, U. Filobello-Niño, R. Castañeda-Sheissa, L. Hernández-Martínez, and A. Sarmiento-Reyes, "Modified HPMS inspired by homotopy continuation methods," Mathematical Problems in Engineering, vol. 2012, Article ID 309123, 19 pages, 2012.

[37] U. Filobello-Nino, H. Vazquez-Leal, R. Castaneda-Sheissa et al., "An approximate solution of blasius equation by using HPM method," Asian Journal of Mathematics and Statistics, vol. 5, pp. 50-59, 2012.

[38] J. Biazar, F. Badpeima, and F. Azimi, "Application of the homotopy perturbation method to ZakharovKuznetsov equations," Computers and Mathematics with Applications, vol. 58, no. 11-12, pp. 2391-2394, 2009.

[39] J. Biazar and H. Ghazvini, "Homotopy perturbation method for solving hyperbolic partial differential equations," Computers and Mathematics with Applications, vol. 56, no. 2, pp. 453-458, 2008.

[40] J. Biazar and H. Ghazvini, "Exact solutions for non-linear Schrödinger equations by He's homotopy perturbation method," Physics Letters A, vol. 366, no. 1-2, pp. 79-84, 2007. 
[41] J. Biazar, H. Ghazvini, and M. Eslami, "He's homotopy perturbation method for systems of integrodifferential equations," Chaos, Solitons and Fractals, vol. 39, no. 3, pp. 1253-1258, 2009.

[42] J. Biazar, M. Eslami, and H. Aminikhah, "Application of homotopy perturbation method for systems of Volterra integral equations of the first kind," Chaos, Solitons and Fractals, vol. 42, no. 5, pp. 3020 3026, 2009.

[43] J. Biazar and H. Ghazvini, "He's homotopy perturbation method for solving systems of Volterra integral equations of the second kind," Chaos, Solitons and Fractals, vol. 39, no. 2, pp. 770-777, 2009.

[44] J. Biazar and H. Ghazvini, "Numerical solution for special non-linear Fredholm integral equation by HPM," Applied Mathematics and Computation, vol. 195, no. 2, pp. 681-687, 2008.

[45] U. Filobello-Nino, H. Vazquez-Leal, Y. Khan et al., “Hpm applied to solve nonlinear circuits: a study case," Applied Mathematical Sciences, vol. 6, no. 85-88, pp. 4331-4344, 2012.

[46] Y. C. Jiao, Y. Yamamoto, C. Dang, and Y. Hao, "An aftertreatment technique for improving the accuracy of Adomian's decomposition method," Computers and Mathematics with Applications, vol. 43, no. 6-7, pp. 783-798, 2002.

[47] M. A. El-Tawil, A. A. Bahnasawi, and A. Abdel-Naby, "Solving Riccati differential equation using Adomian's decomposition method," Applied Mathematics and Computation, vol. 157, no. 2, pp. 503$514,2004$.

[48] S. H. Behiry, H. Hashish, I. L. El-Kalla, and A. Elsaid, "A new algorithm for the decomposition solution of nonlinear differential equations," Computers and Mathematics with Applications, vol. 54, no. 4, pp. 459-466, 2007.

[49] Y.-G. Wang, W.-H. Lin, and N. Liu, "A homotopy perturbation-based method for large deflection of a cantilever beam under a terminal follower force," International Journal for Computational Methods in Engineering Science and Mechanics, vol. 13, pp. 197-201, 2012.

[50] J. H. He, "An elementary introduction to recently developed asymptotic methods and nanomechanics in textile engineering," International Journal of Modern Physics B, vol. 22, no. 21, pp. 3487-3578, 2008.

[51] M. Merdan, A. Gökdoğan, and A. Yildirim, "On the numerical solution of the model for HIV infection of CD4+ T cells," Computers and Mathematics with Applications, vol. 62, no. 1, pp. 118-123, 2011.

[52] G. A. Baker, Essentials of Padé Approximations, Academic Express, London, UK, 1975.

[53] N. H. Sweilam and M. M. Khader, "Exact solutions of some coupled nonlinear partial differential equations using the homotopy perturbation method," Computers and Mathematics with Applications, vol. 58, no. 11-12, pp. 2134-2141, 2009.

[54] S. Momani, G. H. Erjaee, and M. H. Alnasr, "The modified homotopy perturbation method for solving strongly nonlinear oscillators," Computers and Mathematics with Applications, vol. 58, no. 11-12, pp. 2209-2220, 2009.

[55] Y. Khan and N. Faraz, "Application of modified Laplace decomposition method for solving boundary layer equation," Journal of King Saud University, vol. 23, no. 1, pp. 115-119, 2011.

[56] D. Bahuguna, A. Ujlayan, and D. N. Pandey, "A comparative study of numerical methods for solving an integro-differential equation," Computers and Mathematics with Applications, vol. 57, no. 9, pp. 14851493, 2009.

[57] S. Momani and V. S. Ertürk, "Solutions of non-linear oscillators by the modified differential transform method," Computers and Mathematics with Applications, vol. 55, no. 4, pp. 833-842, 2008.

[58] A. Gökdogan, M. Merdan, and A. Yildirim, "The modified algorithm for the differential transform method to solution of Genesio systems," Communications in Nonlinear Science and Numerical Simulation, vol. 17, no. 1, pp. 45-51, 2012.

[59] P. Y. Tsai and C. K. Chen, "An approximate analytic solution of the nonlinear Riccati differential equation," Journal of the Franklin Institute, vol. 347, no. 10, pp. 1850-1862, 2010.

[60] A. E. Ebaid, "A reliable aftertreatment for improving the differential transformation method and its application to nonlinear oscillators with fractional nonlinearities," Communications in Nonlinear Science and Numerical Simulation, vol. 16, no. 1, pp. 528-536, 2011.

[61] W. H. Enright, K. R. Jackson, S. P. Norsett, and P. G. Thomsen, "Interpolants for runge-kutta formulas," ACM Transactions on Mathematical Software, vol. 12, no. 3, pp. 193-218, 1986.

[62] E. Fehlberg, "Klassische runge-kutta-formeln vierter und niedrigerer ordnung mit schrittweiten-kontrolle und ihre anwendung auf waermeleitungsprobleme," Computing, vol. 6, pp. 61-71, 1970.

[63] K. Reif, K. Weinzierl, A. Zell, and R. Unbehauen, "A homotopy approach for nonlinear control synthesis," IEEE Transactions on Automatic Control, vol. 43, no. 9, pp. 1311-1318, 1998.

[64] N. Aslam and A. K. Sunol, "Homotopy continuation based prediction of azeotropy in binary and multicomponent mixtures through equations of state," Physical Chemistry Chemical Physics, vol. 6, no. 9, pp. 2320-2326, 2004. 
[65] F. Kubler and K. Schmedders, "Computing equilibria in stochastic finance economies," Computational Economics, vol. 15, no. 1-2, pp. 145-172, 2000.

[66] R. C. Melville, L. Trajkovic, S. C. Fang, and L. T. Watson, "Artificial parameter homotopy methods for the DC operating point problem," IEEE Transactions on Computer-Aided Design of Integrated Circuits and Systems, vol. 12, no. 6, pp. 861-877, 1993.

[67] K. Yamamura, T. Sekiguchi, and Y. Inoue, "A fixed-point homotopy method for solving modified nodal equations," IEEE Transactions on Circuits and Systems I, vol. 46, no. 6, pp. 654-665, 1999.

[68] R. Geoghegan, J. C. Lagarias, and R. C. Melville, "Threading homotopies and DC operating points of nonlinear circuits," SIAM Journal on Optimization, vol. 9, no. 1, pp. 159-178, 1999.

[69] J. Lee and H. D. Chiang, "Constructive homotopy methods for finding all or multiple dc operating points of nonlinear circuits and systems," IEEE Transactions on Circuits and Systems I, vol. 48, no. 1, pp. 51-66, 2001.

[70] A. Ushida, Y. Yamagami, Y. Nishio, I. Kinouchi, and Y. Inoue, "An efficient algorithm for finding multiple DC solutions based on the SPICE-oriented Newton homotopy method," IEEE Transactions on Computer-Aided Design of Integrated Circuits and Systems, vol. 21, no. 3, pp. 337-348, 2002.

[71] H. Vázquez-Leal, L. Hernández-Martínez, and A. Sarmiento-Reyes, "Double-bounded homotopy for analysing nonlinear resistive circuits," in Proceedings of the IEEE International Symposium on Circuits and Systems (ISCAS '05), pp. 3203-3206, May 2005.

[72] M. Van Barel, K. D. Ikramov, and A. A. Chesnokov, "A continuation method for solving symmetric Toeplitz systems," Computational Mathematics and Mathematical Physics, vol. 48, no. 12, pp. 2126-2139, 2008.

[73] H. Vázquez-Leal, L. Hernández-Martínez, A. Sarmiento-Reyes, and R. Castañeda-Sheissa, "Numerical continuation scheme for tracing the double bounded homotopy for analysing nonlinear circuits," in Proceedings of the International Conference on Communications, Circuits and Systems, pp. 1122-1126, May 2005.

[74] L. Trajkovic, R. C. Melville, and S. C. Fang, "Passivity and no-gain properties establish global convergence of a homotopy method for DC operating points," in Proceedings of the IEEE International Symposium on Circuits and Systems, pp. 914-917, May 1990.

[75] M. M. Green, "Efficient continuation method for use in globally convergent DC circuit simulation," in Proceedings of the 3rd URSI International Symposium on Signals, Systems and Electronics (ISSSE '95), pp. 497-500, October 1995.

[76] L. B. Goldgeisser and M. M. Green, "A method for automatically finding multiple operating points in nonlinear circuits," IEEE Transactions on Circuits and Systems I, vol. 52, no. 4, pp. 776-784, 2005.

[77] D. M. Wolf and S. R. Sanders, "Multiparameter homotopy methods for finding dc operating points of nonlinear circuits," IEEE Transactions on Circuits and Systems I, vol. 43, no. 10, pp. 824-838, 1996.

[78] L. O. Chua and A. Ushida, "A switching-parameter algorithm for finding multiple solutions of nonlinear resistive circuits," International Journal of Circuit Theory and Applications, vol. 4, no. 3, pp. 215-239, 1976.

[79] K. S. Chao, D. K. Liu, and C. T. Pan, "A systematic search method for obtaining multiple solutions of simultaneous nonlinear equations," IEEE Transactions on Circuits and Systems, vol. 22, no. 9, pp. 748$752,1975$.

[80] F. H. Branin Jr., “Widely convergent method for finding multiple solutions of simultaneous nonlinear equations," IBM Journal of Research and Development, vol. 15, no. 9, pp. 2779-2780, 1973.

[81] L. Vandenberghe and J. Vandewalle, "Variable dimension algorithms for solving resistive circuits," International Journal of Circuit Theory and Applications, vol. 18, no. 5, pp. 443-474, 1990.

[82] M. Kojima and Y. Yamamoto, "Variable dimension algorithms: basic theory, interpretations and extensions of some existing methods," Mathematical Programming, vol. 24, no. 2, pp. 177-215, 1982.

[83] Y. Yamamoto, "A variable dimension fixed point algorithm and the orientation of simplices," Mathematical Programming, vol. 30, no. 3, pp. 301-312, 1984.

[84] H. Vázquez-Leal, L. Hernández-Martínez, A. Sarmiento-Reyes, and R. S. Murphy-Arteaga, "Improving multi-parameter homotopy via symbolic analysis techniques for circuit simulation," in Proceedings of the European Conference on Circuit Theory and Design, vol. 2, pp. 402-405, 2003.

[85] J. S. Roychowdhury and R. C. Melville, "Homotopy techniques for obtaining a DC solution of largescale MOS circuits," in Proceedings of the 33rd Annual Design Automation Conference, pp. 286-291, June 1996.

[86] J. Roychowdhury and R. Melville, "Delivering global DC convergence for large mixed-signal circuits via homotopy/continuation methods," IEEE Transactions on Computer-Aided Design of Integrated Circuits and Systems, vol. 25, no. 1, pp. 66-78, 2006. 
[87] L. Trajkovic, R. C. Melville, and S. C. Fang, "Finding DC operating points of transistor circuits using homotopy methods," in Proceedings of the IEEE International Symposium on Circuits and Systems, pp. 758-761, June 1991.

[88] L. Trajkovic and A. N. Willson Jr., "Theory of dc operating points of transistor networks," Archiv fur Elektronik und Ubertragungstechnik, vol. 46, no. 4, pp. 228-241, 1992.

[89] H. Vazquez-Leal, R. Castaneda-Sheissa, A. Y1ldırım et al., "Biparameter homotopybased direct current simulation of multistable circuits," British Journal of Mathematics and Computer Science, vol. 2, no. 3, pp. 137-150, 2012.

[90] Y. Inoue, S. Kusanobu, K. Yamamura, and T. Takahashi, "Newton-fixed-point homotopy method for finding dc operating points of nonlinear circuits," in Proceedings of the International Technical Conference on Circuits/Systems, Computer and Communications (ITC-CSCC '01), vol. 1, pp. 370-373, Tokushima, Japan, July 2001.

[91] L. Trajković and W. Mathis, "Parameter embedding methods for finding dc operating points: formulation and implementation," in Proceedings of the NOLTA, pp. 1159-1164, Dicember 1995.

[92] L. Trajković, R. C. Melville, and S. C. Fang, "Passivity and no-gain properties establish global convergence of a homotopy method for DC operating points," in Proceedings of the IEEE International Symposium on Circuits and Systems, pp. 914-917, May 1990.

[93] H. Vazquez-Leal, L. Hernandez-Martinez, A. Sarmiento-Reyes, R. Castaneda-Sheissa, and A. Gallardo-Del-Angel, "Homotopy method with a formal stop criterion applied to circuit simulation," IEICE Electronics Express, vol. 8, no. 21, pp. 1808-1815, 2011. 


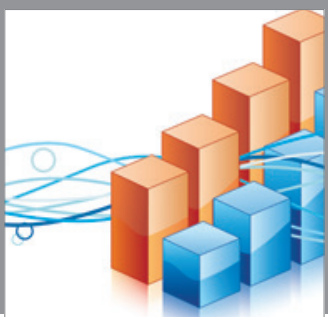

Advances in

Operations Research

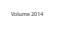

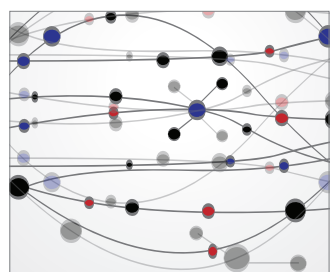

\section{The Scientific} World Journal
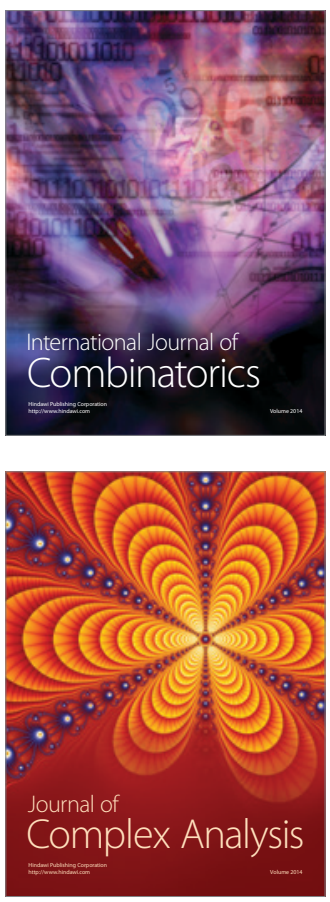

International Journal of

Mathematics and

Mathematical

Sciences
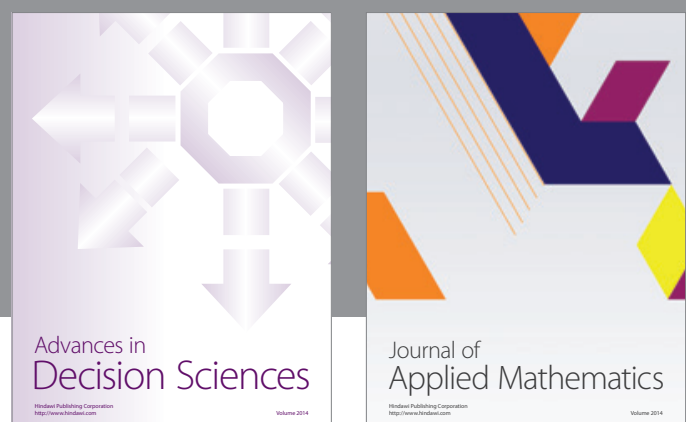

Journal of

Applied Mathematics
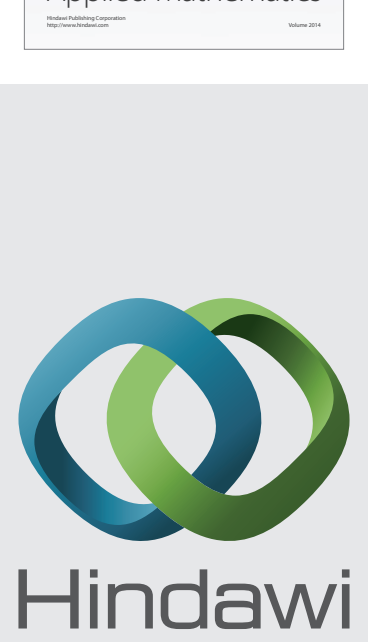

Submit your manuscripts at http://www.hindawi.com
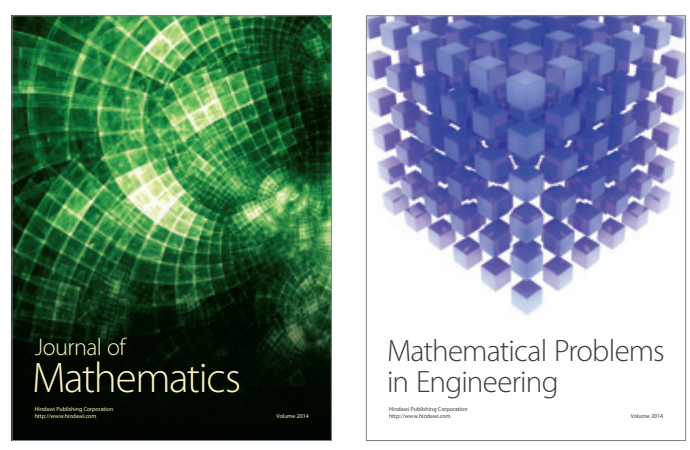

Mathematical Problems in Engineering
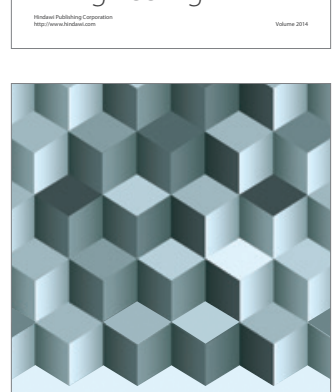

Journal of

Function Spaces
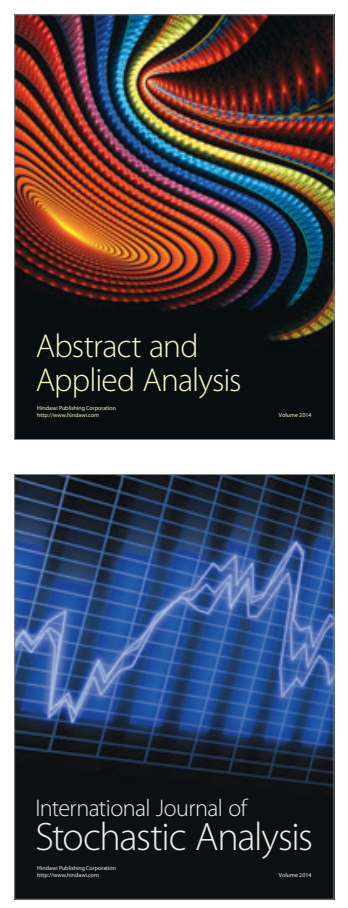

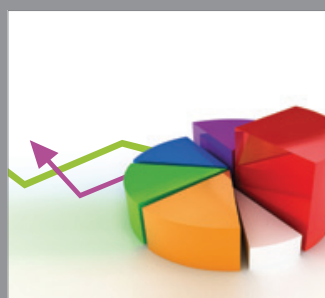

ournal of

Probability and Statistics

Promensencen
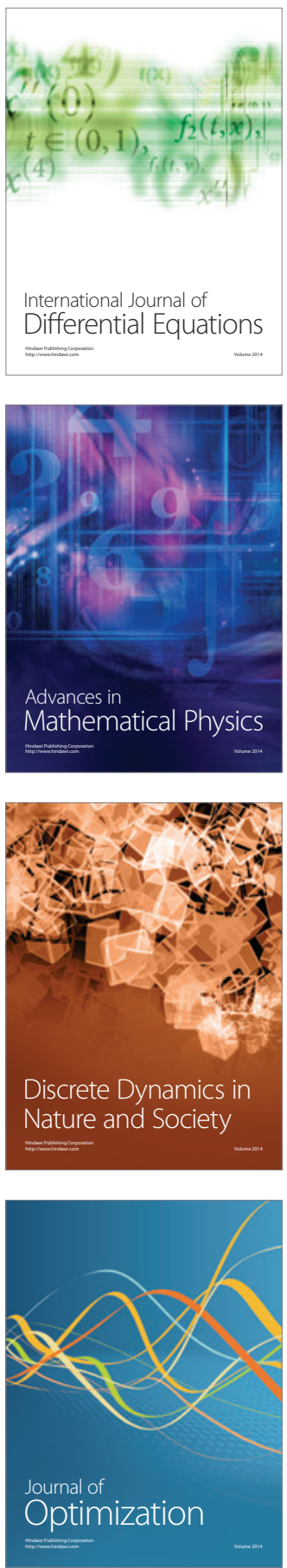